\title{
Quantum Healing - A Novel Current Concept of Holistic Healing
}

\section{Abstract}

Human body works as a whole, has an excellent capacity to heal by itself. Holistic healing is a whole body healing yields better results without adverse effects, inexpensive, unlike reductionist view of healing. Inside and outside of our body consists of energy. Most of all diseases are starts in mind, mind is the canvas, on which our thoughts are projected, is a part of human consciousness. Mind is the holographic representation of the human body. With our positive thoughts, genuine intention to heal known as quantum thinking and healing takes place at spiritual level by producing endorphins. Endorphins are opioid peptides, has activity, anti- aging, and alteration in gene expression , can be used to treat many diseases like cancer, auto-immune diseases, and infectious diseases. This article highlights about current concept of holistic healing is quantum healing and application in treatment of diseases.

Keywords: Anti-Inflammatory; NF-KB; STAT 3; IL-1; IL-6; Endorphins; Cancer; Autoimmune diseases many activities such as immune stimulatory, anti-inflammatory, stress buster

Mini-Review
Volume 10 Issue 2 - 2017

Shrihari TG*
Department of Oral Medicine and Oral Oncology, Krishna
Devaraya College of Dental Sciences and Hospital, India
*Corresponding author: Shrihari TG, Department of Oral
Medicine and Oral Oncology, Krishna Devaraya College of
Dental Sciences and Hospital, Bangalore -562157, Karnataka,
India, Email: drshrihariomr@gmail.com
Received: September 20, 2017 | Published: December 22,
2017

\section{Introduction}

Our modern medicine such as using chemical drug to heal disease by considering our body as bits and pieces, rather than as a whole, has many adverse effects, which is a major cause of death worldwide. Holistic medicine is a whole body healing; Human body has a capacity to heal by itself without any adverse effects, yields better results.

Quantum healing is one of the novels holistic medicine begins to emerge. Matter is not made out of matter, but energy. Matter can be converted in to energy, energy can be converted in to matter is a theory of aduality given by Nobel laureate Hans Peter Duerr. Body tries to rebuilt each time, when it oscillate between energy and particles.

Each healthy cell of our body emits photons by DNA, disease is an altered emission of photons. Inside and outside of our body consists of energy. Photons jumping in and around the body encircling an individual and counter photons may be jumping outside is known as quantum entanglement or quantum tunneling [1-16].

Most of all diseases are starts in mind, ends in mind. With positive thoughts (quantum thinking) in our mind, we can heal any diseases including cancer. Mind is nothing but a consciousness, is a canvas, where our thoughts are projected. Mind is a holographic representation of our body. Quantum field is the total collective consciousness. Inside and outside of human the body consists of energy, by using this energy we can heal any disease including cancer is known as body energy healing or Quantum healing. Positive thoughts with genuine intention to heal known as quantum thinking, healing takes place at spiritual level by producing endorphins [1-16].

Rekhi, Pranic healing, Pranayama, acupuncture, Chi therapy produces endorphins, endorphins are endogenous morphine, secreted by pituitary gland and nervous system. Endorphins are endogenous uploads, neuropeptides, produced by pituitary gland in response to stress and pain through corticotrophic releasing hormone (CRH) release from hypothalamus [2,3]. Stress related altered DNA repair by altering DNA repair enzyme methyl transferase, which is lowered in stressed individuals either by release of free radicals such as ROS, RNS, NO from inflammatory cells (Macrophages, dendritic cells and Neutrophils) and cytokines such as TNF- $\alpha$ or through inactivation of P53 tumor suppressor gene by activating NF-KB key transcription factor involved in tumor progression $[4,5,11,17]$.

Chronic inflammation is considered as a seventh hall mark of cancer. $25 \%$ of all cancers are associated with chronic inflammatory conditions. Some inflammatory conditions or injury that are associated with malignancy are Lichen planus, Oral submucous fibrosis, gingivitis and chronic periodontitis associated oral squamous cell carcinoma, sialadenitis related salivary gland carcinoma, Gastric acid associated Barrett's metaplasia and reflux esophagitis associated esophageal carcinoma, Sjogren's syndrome and Hashimoto's thyroiditis associated mucosa associated lymphoid tissue lymphoma, UV radiation associated skin inflammation melanoma, Silica, asbestosis, smoking associated silicosis and bronchitis associated lung carcinoma, Prostatitis induced prostate carcinoma, chronic pancreatitis induced pancreatic cancer, Hepatitis B induced hepatocellular carcinoma, HPV induced cervical cancer and pharyngeal cancer. Human herpes virus 8 (HHV8) induced Kaposi's sarcoma [6,8].

Chronic inflammatory mediators such as TNF- $\alpha$, IL- 1 ,IL6 release from inflammatory cells such as tumor associated macrophages and neutrophils in tumor microenvironment secrete free radicles activates key transcription factors such as NF-KB and STAT-3 which is involved in tumor progression by cell proliferation (cyclin D,C-MYC, $\mathrm{P}^{21}$ ), cell survival (BCL-2,BCL$\mathrm{XL}$, CFLIP, survivin), angiogenesis (IL-8,VEGF, COX-2), chronic 
inflammation (MHC-1, MHC-11, cytokines), immune suppression (IL-10, TGF- $\beta$, NO), invasion and metastasis (ICAM-1, VCAM1,ELAM-1, upa, E-selectin, MMP-2,9) [6,8].

Endorphins are endogenous morphine, there are three types includes beta- endorphins, enkephalins, and dynorphins operate via $\mu(\mathrm{mu})$, delta , and $\mathrm{k}$ receptors situated on brain, nervous system, immune cells. Beta - endorphin is abundant, potent endorphins synthesized and stored in the anterior pituitary gland, it is a precursor of protein proopiomelanocortin (POMC).

Immune cells produce endorphins at the site of inflammation reducing inflammation by producing cytokines such as IFN-Y, IL10, IL-18. Opioid peptides has affinity for mu, delta, kappa various opioid receptors in inflammatory state, the opioid receptors have been increased in the periphery [7].

It has a natural holistic healer by activating immune cells such as macrophages, NK cells, T and B lymphocytes producing IFN- $\Upsilon$, Opsonin, granzyme -B involved in antiviral, antitumor, antiinflammatory, and apoptotic activity. Reduces stress by reducing cortisol, neuropeptides such as nor- epinephrine through HPA axis (hypothalamo-pituitary adrenal axis), inhibiting activation of sympathetic nervous system of autonomic nervous system, which is involved in anti-inflammatory activity and peripheral immunity. Binding of Beta endorphins to the $\mu(\mathrm{mu})$ receptors in the central nervous system results in inhibiting an inhibitory neurotransmitter GABA and stimulating dopamine release responsible for euphoria, inhibits pain , and tranquility of mind. Binding of Beta - endorphins to $\mu(\mathrm{mu})$ receptors in the peripheral nervous system results in analgesic activity by reducing substance $\mathrm{P}$ a neurotransmitter of pain $[7,12]$.

Anti-inflammatory role by reducing production of cortisol, norepinephrine neuropeptide mediated release of TNF- $\alpha$, IL-1 $\beta$, IL-6, COX-2 pro-inflammatory cytokines and stimulating production of anti-inflammatory cytokines such as IL-2, IL-10, IL-18, and IFN-Y. Tranquility of mind can be obtained to heal disease [16].

Beta- endorphins can be used in treatment of autoimmune diseases by decreasing stress mediated release of cortisol and neuropeptides activates inflammatory mediators such as TNF- $\alpha$, IL-1, IL-6 mediated activation of key transcription factors such as NF-KB and STAT-3 involved in immune modulation, chronic inflammation, conversion of Th1 type lymphocyte to Th2 type, activation of proteolytic enzymes like MMP's (matrix metallo proteases) results in cellular changes and tissue damage $[10,15]$.

Antitumor activity of beta- endorphins by activating immune cells such as NK cells, macrophages, T lymphocytes, produce IFN- $\Upsilon$, opsonin , granzyme $-\mathrm{B}$, has anti-viral , anti-inflammatory activity by reducing inflammatory mediators such as IL- 1 ,TNF- $\alpha$, IL- 6 mediated activation of key transcription factors NF-KB, STAT3 involved in tumor progression, activation of anti-inflammatory cytokines such as IL-10, IL-2, IL-18 and IFN- $\Upsilon$ involved in suppression of inflammation induced cancer [14,15].

Promote and prolong human life span by lengthening telomeres which shorten with aging. Mindfull meditation alters the environment of gene expression [16].

Many studies are conducted on patients with cancer and other disease has shown positive results. We have to understand lot more about current concept of emerging quantum healing and its application in diseases.

\section{Acknowledgment}

None.

\section{Conflict of Interest}

None.

\section{References}

1. Hegde BM (2015) Human mind and quantum healing. Indian academy of clinical medicine 16(3): 182-183.

2. Shrihari TG (2017) Endorphins on cancer: A novel therapeutic approach. J Carcinog Mutagen 8: 298.

3. Shrihari TG (2017) Dual role of inflammatory mediators in cancer. Ecancermedicine 11: 721-730.

4. Zhang, Chang Q (2013) Role of Beta endorphin in control of stress and cancer progression in fetal alcohol exposed rats. Thesis 8: 13.

5. Shrihari TG (2016) Inflammation related cancer -Highlights. J Carcinog Mutagen 7: 269.

6. Stojanovich L (2016) Stress and autoimmunity. Autoimmunity Reviews 9: 271-276.

7. Segerstrom SC, Miller GE (2013) Psychological stress and human immune system. A meta-analytic study of 30 years of inquiry. Psychological Bulletin 130(4): 601-630.

8. Stojanovich L, Marisavijevich D (2008) Stress as a trigger of autoimmune disease. Autoimmun Rev 7(3): 209-213.

9. Zhang C, Murugan S, Boyadjieva N, Jabbar S, Shrivastava P, et al. (2015) Beta endorphin cell therapy for cancer prevention. Cancer prev Res (Phila) 8(1): 56-67.

10. Moreno-smith M, Lutgendorf SK, Sood AK (2010) Impact of stress on cancer metastasis. Future Oncol 6(12): 1863-1881.

11. Ondicova K, Mravec B (2010) Role of nervous system in cancer aetiopathogenesis. The lancet oncology 11(6): 596-601.

12. Lennon FE, Moss J, Singleton PA (2012) The $\mu$ - Opioid receptor in cancer progression: Is there a direct effect. Anesthesiology 116 (4): 940-945.

13. Dowlati Y, Herrmann N, Swardfager W, Liu H, Sham L, et al. (2010) A meta-analysis of cytokines in major depression . Biol psychiatry 67(5): 446-457.

14. Kuebler U, Zuccarella HC, Arpagaus A, Wolf JM, Farahmand F, et al. (2015) Stress induced modulation of NF-KB activation, inflammation - associated gene expression and cytokine levels in blood of healthy men. Brain Behav Immun 46: 87-95.

15. Archana S, Deepali V (2014) Endorphins: Endogenous opioid in human cells. World journal of pharmacy and pharmaceutical sciences 4(1): 357-374.

16. Shrihari TG (2017) Quantum healing approach to new generation of holistic healing. Transl Med 7(3): 198.

17. Iwaszkiewicz KS, Schneider JJ, Hua S (2013) Targeting peripheral opioid receptors to promote analgesis and anti-inflammatory actions. Front pharmacol 24(4): 132. 